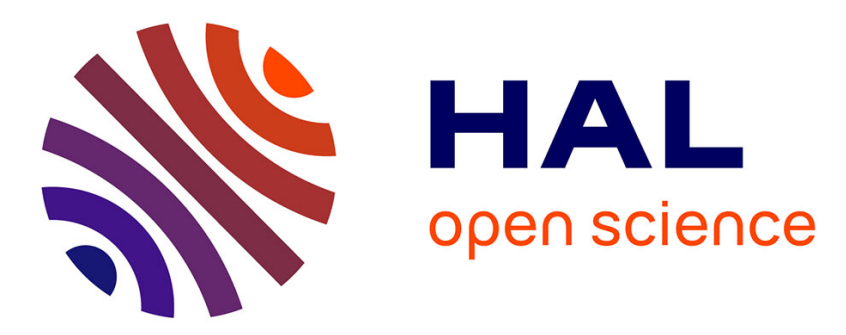

\title{
Raman scattering: measurements of depolarization ratios and order parameters in an oriented nematic mesophase
}

\author{
J.-P. Heger
}

\section{- To cite this version:}

J.-P. Heger. Raman scattering: measurements of depolarization ratios and order parameters in an oriented nematic mesophase. Journal de Physique Lettres, 1975, 36 (9), pp.209-212. 10.1051/jphyslet:01975003609020900 . jpa-00231190

\section{HAL Id: jpa-00231190 https://hal.science/jpa-00231190}

Submitted on 1 Jan 1975

HAL is a multi-disciplinary open access archive for the deposit and dissemination of scientific research documents, whether they are published or not. The documents may come from teaching and research institutions in France or abroad, or from public or private research centers.
L'archive ouverte pluridisciplinaire HAL, est destinée au dépôt et à la diffusion de documents scientifiques de niveau recherche, publiés ou non, émanant des établissements d'enseignement et de recherche français ou étrangers, des laboratoires publics ou privés. 


\title{
RAMAN SCATTERING : MEASUREMENTS OF DEPOLARIZATION RATIOS AND ORDER PARAMETERS IN AN ORIENTED NEMATIC MESOPHASE
}

\author{
J.-P. HEGER \\ Laboratoire de Physique Expérimentale \\ Ecole polytechnique fédérale, Lausanne, Suisse
}

(Reçu le 7 avril 1975, accepté le 21 mai 1975)

\begin{abstract}
Résumé. - Une technique Raman d'origine récente [1, 2] nous a permis d'étudier en fonction de la température l'évolution de deux facteurs de dépolarisation et d'en déduire le comportement des paramètres d'ordre $\left\langle P_{2}\right\rangle$ et $\left\langle P_{4}\right\rangle$ dans le $p$-heptyle- $p^{\prime}$-nitrile biphenyle, sans faire usage d'une sonde non nématique. Les résultats obtenus montrent que si les valeurs de $\left\langle\boldsymbol{P}_{\mathbf{2}}\right\rangle$ ont une allure explicable dans le cadre des théories du champ moyen existantes, celles de $\left\langle\boldsymbol{P}_{4}\right\rangle$ sont notablement inférieures à ce que ces théories permettent de prévoir.
\end{abstract}

Abstract. - A recent Raman investigation technique [1, 2] has been used to study the evolution, with respect to temperature, of two depolarization ratios and deduce the behaviour of the order parameters $\left\langle P_{2}\right\rangle$ and $\left\langle P_{4}\right\rangle$ in the nematic liquid crystal $p$-heptyl-p'-nitrile-biphenyl without recourse to a non-nematic probe. Results show that while $\left\langle\boldsymbol{P}_{\mathbf{2}}\right\rangle$ values can be accounted for within the frame of existing mean-field theories, $\left\langle\boldsymbol{P}_{4}\right\rangle$ values are somewhat lower than what could be predicted by these theories.

1. Introduction. - A recent investigation technique has been proposed [1] and used $[2,3]$ with some success to achieve simultaneous measurements of the order parameters $\left\langle P_{2}\right\rangle$ and $\left\langle P_{4}\right\rangle$ in a macroscopically aligned nematic mesophase by means of Raman scattering. It involved the observation of two depolarization ratios relative to the $2^{\prime} 230 \mathrm{~cm}^{-1}(-\mathrm{C} \equiv \mathrm{N}$ stretching) Raman line emitted by a non-nematic probe molecule : $\quad \mathrm{N}$-( $p^{\prime}$-butoxybenzilidene)-p-cyanoaniline dissolved either in MBBA or in EBBA.

We report here a similar investigation performed on the recently found nematic liquid crystal $p$-heptyl- $p^{\prime}$ nitrile biphenyl (hereafter HNBP for short). The molecules of this compound exhibit a terminal $-\mathrm{C} \equiv \mathrm{N}$ radical located along the major axis, allowing a direct measurement of the environmental changes experiences by the nematic molecules themselves without recourse to an additional probe.

2. Expérimental. - The samples were flat, thin samples of HNBP contained between two square optical glass walls previously rubbed to achieve a macroscopic homogeneous alignment of the nematic liquid crystal enclosed between them. The sample amount of HNBP used had an initial purity of more than $96 \%$. The nematic-to-isotropic liquid transition temperature was found to be $42.2^{\circ} \mathrm{C}$ [4] prior to conducting the Raman investigation. A check of the samples after completion of each series of measurements indicated that in no case had the transition temperature varied significantly.

The sandwich samples of HNBP were mounted in a glass-flow type Raman cell in order to monitor and record the temperature with a precision of $\pm 0.1^{\circ} \mathrm{C}$ and thermal fluctuations equally not greater than $\pm 0.1{ }^{\circ} \mathrm{C}$ during the time necessary to perform each intensity measurement. The plane of the sample was tilted by an angle $\varepsilon=17.5^{\circ}$ with respect to the direction of the incident laser radiation $(z$-axis of the Laboratory Frame, hereafter denoted by LF, see Fig. 1) and the scattered light was observed at $90^{\circ}$ to the direction of the incident laser beam ( $y$-axis of the LF). The plane of the samples could be rotated around an axis of symmetry perpendicular to this very plane, so that the direction of macroscopic alignment of the nematic layer could be rendered either parallel or perpendicular to the scattering plane $(y-z$ plane of the LF). Stokes-shifted Raman records of the $2^{\prime} 230 \mathrm{~cm}^{-1}$ $-\mathrm{C} \equiv \mathrm{N}$ stretching line were performed through a SPEX 1401 Double Monochromator equipped with two 1'200 grooves per $\mathrm{mm}$ gratings used in the first order, and with standard attachments such as a polaroid analyser and a quartz wedge polarization scrambler.

The 5'145 £ emission line of a Spectra Physics Model 165 Argon Ion laser was used as the primary source of 
excitation. The polarization of the incident laser beam was chosen throughout the experiment to be parallel to the scattering plane.

3. Results and discussion. - Considering the geometrical disposition of figure $1 a$ and $b$, one may introduce two depolarization ratios : $\rho_{\mathrm{a}}$ and $\rho_{\mathrm{b}}$, having both the same geometrical definition :

$$
\rho_{\mathrm{a} \mathrm{or} \mathrm{b}}=\frac{I_{y x}}{I_{y z}}
$$

the distinction between them being related only to the fact that the macroscopic alignment direction is chosen either parallel (e. g. Fig. 1 case $(a)$ ) or perpendicular (e. g. case $(b))$ to the $y-z$ scattering plane.
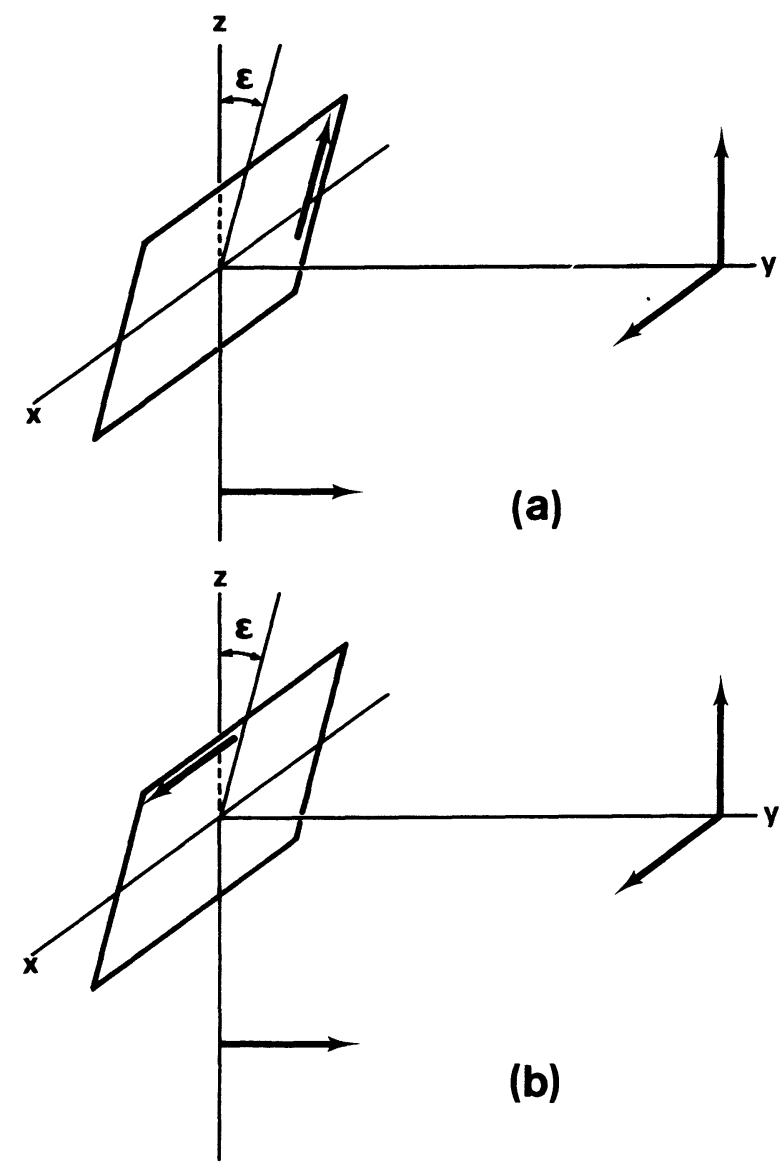

FIG. 1. - Scattering geometry and sample disposition : $z-y$ defines the scattering plane. Case $(a)$ : sample macroscopic alignment direction parallel to the scattering plane, case $(b)$ : macroscopic alignment direction perpendicular to the scattering plane.

A relation can be established between these depolarization ratios and the two order parameters $\left\langle P_{2}\right\rangle$ and $\left\langle P_{4}\right\rangle$ by considering the $Y X$ and $Y Z$ components of a light scattering tensor, in a manner analogous to what has been published formerly by Priestley and Pershan [1]. Following the same treatment one is led to express this dependence by the form :

$$
\begin{aligned}
& <P_{2}>=1-\frac{\frac{3}{2} \rho_{\mathrm{a}}\left(\rho_{\mathrm{b}}+4\right) E}{4\left(A-\rho_{\mathrm{a}} C\right)+\left(\rho_{\mathrm{b}}+4\right)\left(B-\rho_{\mathrm{a}} D\right)} \\
& \left.<P_{4}\right\rangle=1-\frac{\frac{3}{2} \rho_{\mathrm{a}}\left(2 \rho_{\mathrm{b}}+1\right) E}{4\left(A-\rho_{\mathrm{a}} C\right)+\left(\rho_{\mathrm{b}}+4\right)\left(B-\rho_{\mathrm{a}} D\right)}
\end{aligned}
$$

where $A, B, C, D, E$ are numerical factors depending only on the angle of tilt $\varepsilon$ and reflecting the influence of the adopted geometry on the measurements.

In order to check the effects of multiple scattering in the nematic medium, measurements of $\rho_{\mathrm{a}}$ and $\rho_{\mathrm{b}}$ were performed over several samples of increasing thicknesses, i. e. about 50, 75, 100 and $200 \mu$. According to reference [2], a comparison of values of $\rho_{\mathrm{a}}$ and $\rho_{\mathrm{b}}$ so obtained for decreasing thicknesses and extrapolation to zero should lead to limit values of both depolarization ratios.

However, although attempted, this procedure was not conclusive in the present geometry, mainly because of a significant increment in the dispersion of experimental values with decreasing thicknesses.

A choice was made to concentrate on $\rho_{\mathrm{a}}$ and $\rho_{\mathrm{b}}$ values obtained on the $100 \mu$-thick samples because of a still reasonable average accuracy of $\pm 10 \%$ which could be produced over the entire temperature range under examination. Values of $\rho_{\mathrm{a}}$ and $\rho_{\mathrm{b}}$ calculated by use of the integrated intensities constituted raw data to which a least-mean-squares fit was applied. Furthermore, an optical correction was introduced in order to meet the physical requirements imposed on the radiation reemitted by a dipole situated in an uniaxial medium. The need for such a correction originates from the fact that the entrance optical assembly of the spectrometer collects the light reemitted by the scattering medium over large solid angles. Accordingly, account has to be taken of the boundary conditions at

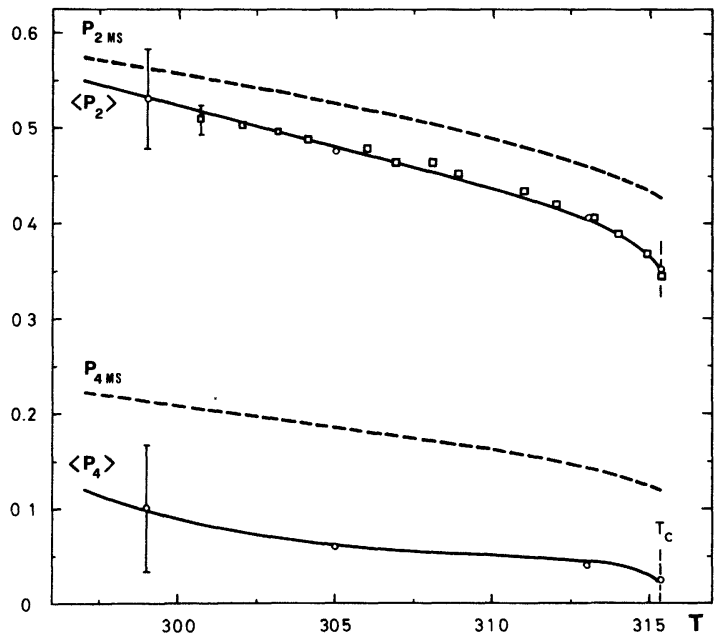

Fig. 2. - Least-mean-squares fit to $\left\langle P_{2}\right\rangle$ and $\left\langle P_{4}\right\rangle$ values vs. absolute temperature for a $100 \mu$-thick HNBP sample. Squares account for $\left\langle P_{2}\right\rangle$ values found using birefringence measurements (see Fig. 3). Dashed curves are Maier and Saupe's prediction for $\left\langle\boldsymbol{P}_{2}\right\rangle$ and $\left\langle\boldsymbol{P}_{4}\right\rangle$. 
the nematic-glass interface in order to obtain proper values of the depolarization ratios $[1,2]$.

Figure 2 shows the results given by this calculation on the values of $\rho_{\mathrm{a}}$ and $\rho_{\mathrm{b}}$. A plot of $\left\langle P_{2}\right\rangle$ and $\left\langle P_{4}\right\rangle$ obtained through use of eq. (1) and (2) vs. absolute temperature has been represented together with the theoretical prediction of Maier and Saupe [5].

It is nevertheless noteworthy that although the estimate of $\left\langle P_{2}\right\rangle$ values differs noticeably from what can be deduced from the Maier and Saupe theory, it can be favourably compared with results on other nematic mesophases obtained by resonance techniques $[8,9]$. The transition value for $\left\langle P_{2}\right\rangle$ was found to be 0.35 , which, to our knowledge, has not yet been confirmed by other investigations on HNBP up to now.

However, a simple means to check the behaviour of $\left\langle P_{2}\right\rangle$ deduced from Raman depolarization ratios is available through measurements of the birefringence of the nematic medium under investigation [10]. The prism method which has been used earlier by other workers [11-13] proved particularly precise and straightforward. We used in our case a prism constituted by two flat optical glass plates making an angle of $2^{\circ} 14^{\prime} 32^{\prime \prime}$. Macroscopic alignment of HNBP contained in the wedge was, too, achieved by rubbing and measurements of both refractive indices and birefringence were performed at $\lambda=0.5145 \mu$. HNBP acts as a positive uniaxial medium in its nematic phase. Figure 3 presents a plot of the birefringence :

$$
\Delta n=n_{\mathrm{e}}-n_{0}
$$

vs. reduced temperature : $\tau=\left(T-T_{c}\right) / T_{c}$ where $T_{c}$ is the absolute temperature of the nematic - to - isotropic transition.

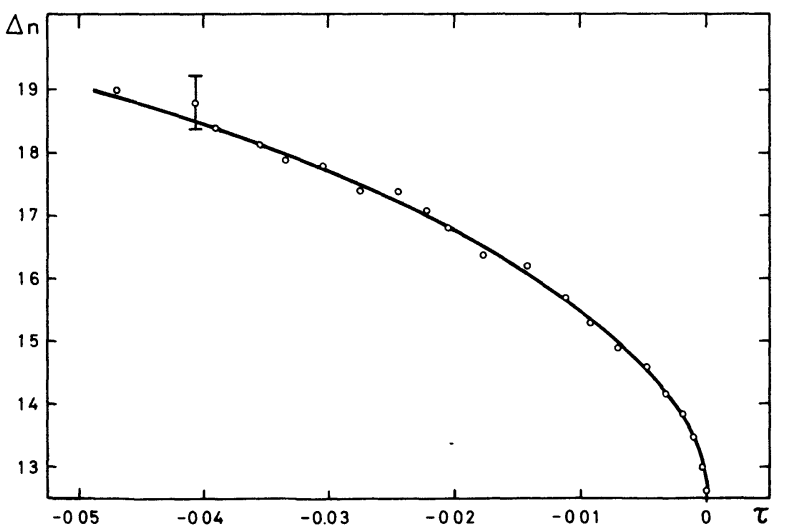

Fig. 3. - Birefringence of HNBP vs. reduced temperature, $\tau=\left(T-T_{c}\right) / T_{c}$ at $\lambda=0.5145 \mu$.

The order parameter $\left\langle P_{2}\right\rangle$ is then known to obey a relationship :

$$
<P_{2}>\propto d^{-1} \Delta n
$$

where $d$ is the specific gravity of the nematic compound investigated [14]. Knowledge of the refractive indices $n_{\mathrm{e}}$ and $n_{0}$ allows a precise determination of the proportionality, an almost temperature-independent, factor between $\left\langle P_{2}\right\rangle$ and $d^{-1} \Delta n$ in (3). This factor was found to be 2.6593 in our case. $\left\langle P_{2}\right\rangle$ values obtained in this mannear appear as squares on figure 2 and give good support to the numerical values of $\left\langle P_{2}\right\rangle$ deduced from Raman measurements.

Calculations performed on the molecular field model proposed by Humphries, James and Luckhurst [6] indicated that our results on $\left\langle\boldsymbol{P}_{\mathbf{2}}\right\rangle$ can be reasonably well fitted by giving the two H. J. L. extra free parameters the values $\lambda=-0.34$ and $\gamma=4.9$. However, neither Maier and Saupe, nor Humphries, James and Luckhurst models would account for the experimental behaviour of $\left\langle P_{4}\right\rangle$ as it appears on figure 2 . Two important features can be extracted out of this plot :

- experimental values of $\left\langle P_{4}\right\rangle$ are definitely lower than what can be deduced from both theories,

- an upward curvature of the $\left\langle P_{4}\right\rangle$ curve appears for temperatures below $10^{\circ} \mathrm{C}$ from the nematic-to-isotropic liquid transition.

The first point has already been pointed out by other workers [2,3] who were able to demonstrate negative values for $\left\langle P_{4}\right\rangle$ in the nematic phase of either MBBA or EBBA. A tentative hypothesis was then emitted to question either the unicity of the choice of the order parameters, in view of the flexibility of part of the investigated molecule, or to assume the possibility of pair correlations between adjacent molecules which would lead in turn to the inapplicability of a mean field theory. In the case of HNBP, the flexible part of the molecule should be the heptyl tail in place of a butyl tail as happens in the MBBA or EBBA molecules. The fact that the calculated values of $\left\langle P_{4}\right\rangle$ do not exhibit a definite trend toward negative values up to the transition temperature would incline us to consider the influence of side movements of the heptyl tail as less important here than in the case of MBBA or EBBA. Further studies on related nematic molecules should give a better insight on this point.

It is not clear at the present time whether the abovementioned upward curvature on the $\left\langle P_{4}\right\rangle$ plot appears merely as a side-effect of the treatment of the experimental depolarization ratios or whether an intrinsic property of the nematic phase of HNBP has been so disclosed. Assuming the first hypothesis, the dual dependence of $\left\langle P_{2}\right\rangle$ and $\left\langle P_{4}\right\rangle$ on both depolarization ratios should betray an analogous behaviour, even if slightly, in the values of $\left\langle P_{2}\right\rangle$, which does not seem to be the case. On the other hand, one has to consider the fact that the relations (1) and (2) between the order parameters and the depolarization ratios are obtained by the treatment of a singlemolecule model, while the experimental conditions in which the measurements were performed make it disputable whether the requirements of such a model are fulfilled. In particular, it could be reasonably argued 
that data collected on samples of finite thickness should be impaired for two main reasons :

1) the effect of multiple scattering in the bulk of the nematic layer submitted to irradiation.

2) the influence of molecular interactions acting on the $-\mathrm{C} \equiv \mathrm{N}$ radical under investigation.

As has been mentioned above, the experimental conditions were so chosen as to provide data on samples as thin as possible, while maintaining a tolerable accuracy in the measurements of scattered intensities. It can be assumed by this means that effects of multiple scattering were minimized if not eliminated.

The question about the possible influence of molecular interactions on the actual vibration of the $-\mathrm{C} \equiv \mathrm{N}$ radical in the nematic phase under examina- tion can be partly ansvered by remarking that this vibration is related to a totally symmetric mode in the molecule of HNBP. Past calculations [7] on Raman absolute cross sections in liquid benzene and toluene give support to the fact that totally symmetric modes are but feebly affected by molecular interactions in the liquid state. That it should be true in a nematic mesophase has been an underlying hypothesis in our calculations, but still remains to be studied carefully by appropriate experimental means.

Aknowledgment. - We express our best thanks to Mrs M. Bresse of the Chemistry Division. Laboratory for Electronics and Technology of Informatics, C. E. N., Grenoble, who kindly provided us the amount of HNBP necessary for this work.

\section{References}

[1] Priestley, E. B. and Pershan, P. S., Mol. Cryst. Liq. Cryst. 23 (1973) 369.

[1] Ghen Jen, Clark, N. A., Pershan, P. S. and Priestley, E. B., Phys. Rev. Lett. 31 (1973) 1552.

[3] Priestley, E. B. and Shen Jen, Communication to the Vth International Liquid Crystal Conference, June 17 th-21st (1974) Stockholm.

[4] It should be noted that, owing to the impurities contained in the sample, a difference of $0.4^{\circ} \mathrm{C}$ was found between $T_{c}$ values obtained while raising or decreasing temperature. Each value was however reproducible. Consistently, the numerical values of the depolarization ratios which were used for later calculations are those ones which were obtained while going from the nematic mesophase to the isotropic liquid phase.

[5] Maier, W. and SAUPE, A., Z. Naturforsch. 14a (1959) 882 and 15a (1960) 287.
[6] Humphries, R. L, James, P. G. and LuCKhURST, G. R., J. Chem. Soc., Faraday Trans. 268 (1972) 1031.

[7] Eckhardt, G. and Wagner, W. G., J. Mol. Spectros. 19 (1966) 407.

[8] Rowell, J. C., Phillips, W. D., Melby, L. R. and Panar, M., J. Chem. Phys. 43 (1965) 3442.

[9] Chen, D. H., James, P. G. and Luckhurst, G. R., Mol. Cryst. Liq. Cryst. 8 (1969) 71.

[10] Chandrasekhar, S., Krishanmurti, D. and MadhusuDana, N. V., Mol. Cryst. Liq. Cryst. 8 (1969) 45.

[11] Pellet, O. and Chatelain, P., Bull. Soc. Fr. Mineral 73 (1950) 154

[12] Chatelain, P., ibid. 78 (1955) 262.

[13] Haller, I., Huggins, H. A. and Freiser, M. J., Mol. Cryst. Liq. Cryst. 16 (1972) 53.

[14] Poggi, Y., Labrunie, G. and Robert, s., C. R. Hebd. Séan. Acad. Sci. B 277 (1973) 561. 